\title{
Design and Implementation of Mobile Workflow Function Based on Power Grid Inspection Scenario
}

\author{
Gang Wang, 2,a , Weimin Tang ${ }^{3}$, Haiping Shen ${ }^{3}$,Yu hai ${ }^{1,2}$, Xingchuan Bao ${ }^{1,2}$, Min Xu ${ }^{1,2}$, \\ Zhansheng Hou ${ }^{1,2}$, Haiyun Han ${ }^{1,2}$,Lin Peng ${ }^{1,2}$ \\ ${ }^{1}$ GLOBAL ENERGY INTERCONNECTION RESEARCH INSTITUTE Co., Ltd,Nanjing, Jiangsu, 210003, \\ China \\ ${ }^{2}$ State Grid Key Laboratory of Information \& Network Security, Nanjing,210003, China \\ ${ }^{3}$ State Grid Wuxi Power Supply Company, Wuxi, 214000, China \\ awanggang@geiri.sgcc.com.cn
}

Keywords: power grid inspection scenario, mobile workflow, power mobile operation.

\begin{abstract}
In power grid inspection operations, there is an urgent need for mobile workflows based on power scenarios for on-site process management. This paper studies the workflow engine technology for power grid inspection operations, and designs a simple and easy-to-use mobile workflow architecture to guide on-site inspections for the workflow application scenarios and augmented reality technologies under different operating scenarios of power grid power distribution and power distribution. Operators perform workflows quickly and accurately based on augmented reality technology. The workflow execution client is designed to work collaboratively with the power mobile job client state manager to manage the workflow task through long connection and SMS methods, so as to avoid the problem that the mobile workflow cannot maintain the operation in poor communication conditions, and the power mobile job client state management The implements and workflow process executors work together to manage the mobile workflow tasks and implement a mobile workflow based on the power grid inspection operation scenario.
\end{abstract}

\section{Introduction}

At present, there are many problems in the power grid inspection operations: a wide variety of work instructions and inconvenient on-site use. In the daily guide card processing, paper-based records are used for transmission. This model has many drawbacks, such as the inability to the power grid patrol operations are managed. Problems encountered at the site need to be communicated by telephone. There are many unexpected situations encountered at the operation site and expert help is needed. Therefore, mobile process tools are needed to solve the problems encountered during the patrol operations. With the maturity of augmented reality and mobile technologies and the increasingly powerful processing capabilities of mobile terminals, it is necessary to study a mobile workflow design method based on power grid inspection work scenes to meet the needs of power grid inspection operations.

Considering that many transmission line patrols are conducted in remote mountainous areas, the communication speed is low, and high-availability of power mobile job site processes is required. The mobile work function is complex and needs to move the workflow tool based on the power grid inspection work scene. At present, most workflow processing software only supports the office network and lacks support for mobile job communication. Therefore, it is particularly important to provide a mobile workflow design method based on grid inspection work scenes.

Traditional workflow software includes workflow definition, workflow driver, and workflow management. However, it is not suitable for mobile environments. It is particularly important to provide a mobile workflow design method based on grid inspection task scenarios. 


\section{Mobile workflow application analysis}

With the maturity of mobile technologies and the increasingly powerful processing capabilities of mobile terminals, along with the development of mobile communication technologies, more and more enterprises have expanded their services to mobile, and integrating mobile devices into corporate services has also become an important industry trend. Mobile applications have gradually become the user's preferred mode of interaction, and make full use of the capabilities provided by mobile devices, becoming important business channels and new business model innovations ${ }^{[1-2]}$. Under such technology and business development trends, the mobile platform has become the basic platform for enterprise IT construction. It can help companies quickly realize business mobility, cater to technological trends, adapt to rapid business changes, and accelerate business transformation $^{[3]}$. There are certain applications in the application of power grid patrol operations, such as the application of equipment inspections and equipment testing in the production-related business, and applications such as mobile meter reading and electricity inspection in the marketing business $^{[4]}$. The material warehouse mobile operation application business covers the on-site handover, acceptance, storage, delivery management, as well as the receipt of goods in the warehouse, group shelves, picking off the shelf, delivery .

Most of the workflow management applications in power grid inspection applications are coupled with services. There are not many workflow applications. Most of the work flow tasks are exported offline and cannot support the workflow in real time.

The Mobile Workflow function provides a mobile client remote access application platform. Users can adapt to the business resources in the outdoor environment under the conditions of the security certification. The remote work process assists in the convenient completion of on-site work operations and provides the resources needed for on-site operations ${ }^{[5]}$. The background server-side workflow module is mainly responsible for managing mobile client authentication, providing workflow-driven, management, and other functions. Collaborate with other modules to complete on-site video and voice information exchanges and support remote expert assistance.

\section{Mobile workflow architecture design}

The design method of operation work for grid site operations mainly involves intelligent power patrol application scenarios. A patrol work task is created by maintaining a patrol standardization work instruction card and a patrol cycle, and is pushed to the mobile terminal. The operative receives the task at the mobile terminal, performs a task operation at the job site, and transmits the task result and the record through the mobile terminal device. Back to the server, the server performs statistical analysis. The working mode designed by this method can provide more guidance auxiliary information in real time during the power inspection process. According to the classification information of the power grid site, it can effectively carry out the transmission of power grid operation information and effectively support the power on-site operation work. Include the following steps:

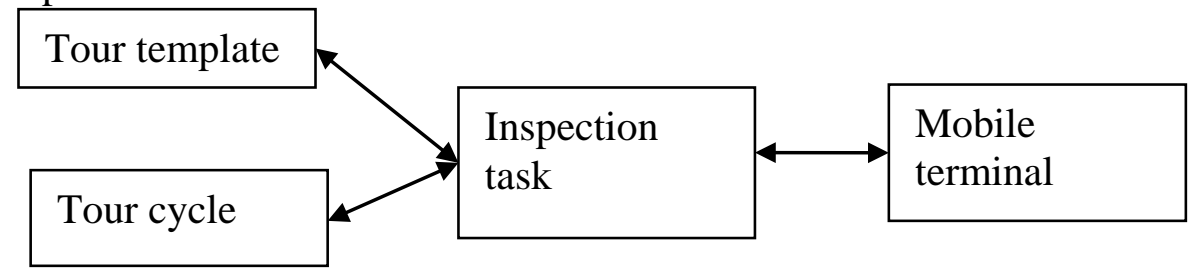

Fig.1Grid patrol operation flow chart

Establish a patrol operation standardization guide card as a template for the new inspection task. At the same time, the cycle management of the inspection task is performed. The patrol cycle and the inspection template are associated with each other to establish a patrol task and pushed to the mobile terminal. The staff receives the task and performs the operation at the site. In the on-site operation process, the staff can realize real-time transmission through video and audio, realize 
remote consultation, strengthen real-time monitoring, and take pictures, upload audio files, and record the inspection results.

The definition of the patrol template stipulates the patrol contents determined by the patrolling location (substation) and patrol type (normal patrol inspection, special patrol inspection, professional patrol inspection, comprehensive patrol inspection, and light out inspection), including patrol preparation stage and patrol implementation stage. And the end of the inspection, and the specific operation steps include the work content, precautions, equipment, loops, executors and so on.

The definition of patrol cycle stipulates that different patrol types in the same substation can establish different patrol cycles. The cycle units can be days, weeks, and months. Each patrol cycle can be associated with a patrol template. The patrol cycle and patrol template can be used to generate the system. The task of inspection work during the corresponding time.

According to the contents of the tour template to establish patrol work tasks, including the task content, patrol team group, patrol date, responsible person, patrol detailed operation steps, step performers, etc. After the completion of the task is released, you can push the operation task from the server directly to Mobile terminals allow users to conveniently receive tasks and implement them.

After the mobile terminal receives the work task on the mobile terminal, it performs on-site patrol tasks, such as visiting the substation, uploading a photo, recording a file, recording the defect content, and transmitting it back to the server's standardized operation management system. The operation task function in the operation management system will be the terminal. Return the data for statistical analysis, generate inspection reports and defect reports.

In order to meet the needs of existing on-site mobile workflow technologies, mobile workflow platforms need to meet the requirements of heterogeneous network access, mobile client processing workflow, provide mobile client authentication, workflow engine driver, workflow definition to meet the needs of mobile operations, Workflow management for mobile job requirements. Design a mobile work flow based on the power grid inspection work scene. The following technical solutions are adopted: Workflow process definer defines a mobile workflow, workflow process executor executes a mobile workflow, workflow execution client is mainly on the power mobile work terminal, accepts execution workflow, feedback workflow status, power movement Job Client Status Manager manages workflow execution client status.

The power mobile client state manager manages the workflow in two ways. The first one is to manage the workflow through the TCP long connection management method to perform the client token connection. Through the token unique status, the workflow is monitored and the client task status is executed. Synchronize to the workflow process executor. The second method is to analyze the workflow execution client message by SMS, extract the mobile work task, and synchronize to the workflow process executor.

The workflow execution client accepts the execution workflow in two ways. In the first type, a TCP long connection is used to create a task task that uniquely identifies the token and accepts the user to perform the task. The second method is to parse the message of the power mobile client status server through SMS and accept the user to perform the task.

Workflow process definers can define parallel, serial move workflows, and define workflow execution roles. The workflow process executor is responsible for executing the workflow defined by the workflow process definer, and the power mobile job client state manager and the workflow execution client drive the process operation.

\section{Functional System Design and Implementation}

For the function of the mobile workflow based on power scenarios, the system must provide functions such as patrol tasks, mobile terminals, task processing, and result feedback, as shown in the following figure. In order to ensure the stable and reliable communication, it is necessary to design modules for mobile work flow client management, mobile workflow drive, work flow processing, and workflow management. These are all completed by a mobile workflow engine. The 
mobile workflow engine is the key to designing and implementing a mobile workflow platform. It is also a difficult design point. Compared with an ordinary workflow, the biggest difference in the mobile workflow is the outdoor communication of information. The conditions are poor, and the workflow is required to maintain the real-time online status to complete the task. Mobile workflow communication needs to be based on 2G/3G/4G networks and completes information transmission through SMS and wireless networks. The following is the implementation process of the power grid inspection process.

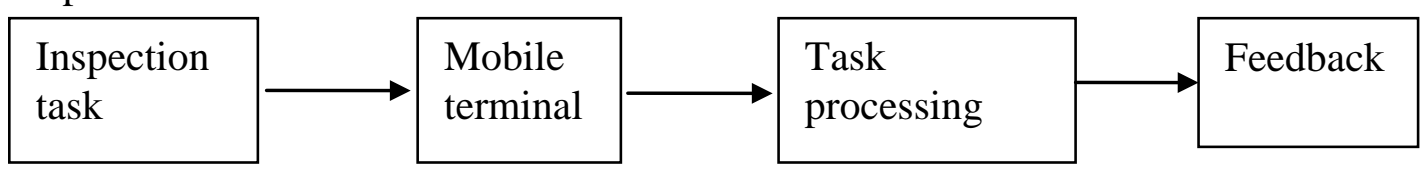

Fig.2Grid patrol process implementation process diagram

\section{Conclusion}

This paper adopts the work flow of tour template, patrol cycle, patrol work task, mobile terminal workflow architecture design, patrol work task, mobile terminal, task processing, result feedback, etc., to enhance the patrol workflow operability and reduce the intensity of on-site staff. Through paperless, pen-less business processing, it is convenient for on-site workers to manage and control the entire process and safety of the site. In order to provide mobile process stability, the workflow execution client cooperates with the power mobile job client state manager to manage workflow tasks through long connection and SMS methods, which prevents the mobile workflow from being unable to maintain operational problems in poor communication conditions. The job client state manager and workflow process executor work together to manage the mobile workflow tasks and achieve a stable transmission workflow.

\section{Acknowledgments}

This work was financially supported by the science and technology project to State Grid Jiangsu Electric Power Corporation 'Research and Application of Augmented Reality Patrol Assistant System Based on Smart Wearable Devices'.

\section{Reference}

[1] Leymann F. Workflow-based Applications. IBM Systems Journal,1997,36(1):102 122.

[2] Eivind Gravdal. Augmented Reality and Object Tracking for Mobile Devices [D]. Norway: Norwegian University,2012:1.

[3] Zhang Hua, Wang Hao. Research and Implementation of Service-Oriented Workflow Compensation Mechanism[J]. Journal of Southeast University: Natural Science Edition. 2009, 39(1): 40-46.

[4] Zhiyong L. Relation-based lightweight workflow engine[J]. Computer Applications and Software, 2005, 4: 053.

[5] Huynh, Duy, Karthik Raveendran, et al. Art of Defense: A Collaborative Handheld Augmented Reality Board Game[C].2009 ACM SIGGRAPH Symposium on Video Games. ACM, 2009,135-142. 